\title{
Correlation of Blood Lead Levels and Elevated Blood Pressure in Newly Detected Hypertensive Patients
}

\author{
Shuaib Ahmed M. A ${ }^{1}$ Raghav Sharma², Shama Prakash K. ${ }^{3}$ \\ 1,2,3 Department of General Medicine, K.S. Hegde Medical Academy, \\ Deralakatte, Mangalore, Karnataka, India.
}

\section{ABSTRACT}

\section{BACKGROUND}

Hypertension is one of the most common diseases all over the world. Lead is a common environmental and industrial pollutant with no beneficial biological role. The contribution of environmental lead exposure to hypertension is an important public health concern. The objective of this study was to determine the blood lead levels in hypertensive patients and to investigate the correlation between blood lead $(\mathrm{B}-\mathrm{Pb})$ levels and the values of blood pressure in hypertensive patients.

\section{METHODS}

This is a hospital based analytical cross-sectional study conducted over a period of one and half years. Subjects included 50 newly detected hypertensive and 50 healthy subjects recruited who were both age and sex matched. Mean of age, blood lead levels, systolic blood pressure (SBP), diastolic blood pressure (DBP) with standard deviation were assessed. Linear regression was used to estimate the predicted systolic blood pressure (BP) and diastolic BP with lead levels. Pearson correlation was used to estimate the correlation between blood lead and blood pressure levels.

\section{RESULTS}

In this study, $52 \%$ of cases and controls were in the age group of 41 - 60 years. $22 \%$ of the subjects were more than 61 years of age. $45 \%$ of the total subjects were females and $55 \%$ were males. $46 \%$ of the controls and $44 \%$ of the cases were females. Comparison of the serum lead levels ( $\mathrm{mcg} / \mathrm{dl}$ ) between cases and controls showed that serum lead levels ( $\mathrm{mcg} / \mathrm{dl}$ ) were higher in Cases group with a t-value of -7.38 and was statistically significant with a P-value of $<0.001$. Comparison of the serum lead levels ( $\mathrm{mcg} / \mathrm{dl}$ ) between the males and females showed that serum lead levels $(\mathrm{mcg} / \mathrm{dl}$ ) were higher in female group with a t-value of -0.151 and was statistically non-significant with a P-value of 0.881 . This study showed that there is an increase in blood lead levels in hypertensive patients compared to the control group. Stage 2 hypertension has higher serum lead level value of 21.228 compared to stage 1 . Serum lead levels were higher in females compared to males.

\section{CONCLUSIONS}

Our study indicated that blood lead level is significantly and positively correlated to blood pressure among newly detected hypertensive patients. Increase in blood lead levels causes increase in both systolic and diastolic blood pressures.

\section{KEY WORDS}

Hypertension, Lead, Systolic Blood Pressure, Diastolic Blood Pressure
Corresponding Author: Dr. Shuaib Ahmed M. A Department of General Medicine, K.S. Hegde Medical Academy, Deralakatte, Mangalore, Karnataka, India., E-mail: shuaib9800@hotmail.com

\section{DOI: $10.14260 /$ jemds/2021/203}

How to Cite This Article: Ahmed MAS, Sharma R, Prakash KS Correlation of blood lead levels and elevated blood pressure in newly detected hypertensive patients. J Evolution Med Dent Sci 2021;10(13):941-946, DOI: $10.14260 /$ jemds $/ 2021 / 203$

Submission 26-11-2020,

Peer Review 30-01-2021,

Acceptance 06-02-2021,

Published 29-03-2021.

Copyright (C) 2021 Shuaib Ahmed M. A., et al This is an open access article distributed under Creative Commons Attribution License [Attribution 4.0 International (CC $B Y 4.0)]$ 


\section{BACKGROUND}

Hypertension is one of the most common diseases worldwide. Due to high blood pressure around 7.6 million deaths (13 - 15 $\%$ of the total) and 92 million disabilities worldwide were attributed in 2001. Hypertension increases the risk of cardiovascular diseases, including coronary heart disease (CHD), congestive heart failure (CHF), ischemic and haemorrhagic stroke, renal failure and peripheral arterial disease. Even though antihypertensive therapy clearly reduces the risks of cardiovascular and renal disease, large segments of the hypertensive population are still either untreated or inadequately treated. ${ }^{1}$

Lead is a highly poisonous metal with no much beneficial biological role. It has been detected in all phases of environment and biological system. The prolonged existence of lead in the blood of humans and animals and its associated health risk, is a topic of current debate and concern. ${ }^{2}$ Moreover, rapid industrialisation and the continued use of leaded gasoline in the developing world appear to be a cause for increasing lead exposure. ${ }^{3}$

Lot of attention has been paid to the probability that lead exposure in the general population among adults can elevate blood pressure and increase the risk for cardiovascular diseases' mortality and morbidity. ${ }^{4}$ There is compelling evidence for this association from the epidemiological literatures but the exact causal nature of the relationship remains controversial. The relationship between lead exposure and the rise of blood pressure has received a great deal of attention as it was implicated that by reducing lead levels in the environment the mortality from cardiovascular diseases might be reduced.

Although the evidence in support of a role of lead induced hypertension is strong, it is not conclusive, nor is the mechanisms by which lead may act on the vascular system is clear. Lead exposure may not be apparent in the general population but may be more of a risk factor for certain "susceptible populations". Hypertension remains a significant risk factor for other forms of cardiovascular diseases; the contribution of potential environmental lead exposure to this risk is an important public health concern. ${ }^{2}$

Keeping this in background, the current study is being conducted to determine the blood lead (B-Pb) levels in hypertensive patients and to investigate the correlation between $\mathrm{B}-\mathrm{Pb}$ levels and the values of blood pressure in hypertensive patients.

\section{METHODS}

This is an analytical hospital based cross sectional study conducted at Justice K.S. Hegde Charitable Hospital, attached to K.S. Hegde Medical Academy, a unit of Nitte University, Deralakatte, Mangalore. This study was conducted for a period of 18 months from January 2015 to June 2016.

\section{Sample Size}

1. Disease group - 50

2. Comparison group -50
Sample size was taken based on the convenience of the study.

\section{Disease Group}

Patients were categorised as cases if the following criteria were met -

1. Systolic blood pressure of $140 \mathrm{~mm} \mathrm{Hg}$ or higher.

2. Diastolic blood pressure of $90 \mathrm{~mm} \mathrm{Hg}$ or higher.

All consenting newly detected hypertensives above 18 years of age coming to Justice K.S. Hegde Charitable Hospital were included in the study.

\section{Exclusion Criteria}

1. Chronic kidney disease.

2. Chronic liver disease.

3. Painter.

4. Hypertensive patients on medication.

\section{Comparative Group}

50 healthy subjects, age and sex matched were taken as controls for this study.

\section{Method of Collection of Data}

In this study, 50 newly detected hypertensive patients were recruited during their visit to K.S. Hegde Charitable Hospital, during January 2015 - June 2016.

After approval by the ethics committee of the K.S. Hegde Charitable Medical Academy, written informed consent was taken prior to the investigation after detailed information was given to the participants regarding the study. 50 healthy age and sex matched volunteers were used as controls.

Each patient was subjected to full complete medical history including duration of the disease. All participants underwent careful physical examination and necessary laboratory investigations to exclude any conditions that may interfere with the disease.

A mean of 3 systolic and diastolic blood pressure measurements in sitting posture was taken. Patients were categorised as hypertensive if any of the following criteria was met-

1. Systolic blood pressure of $140 \mathrm{~mm} \mathrm{Hg}$ or higher,

2. Diastolic blood pressure of $90 \mathrm{~mm} \mathrm{Hg}$ or higher.

\section{Lead Analysis in the Whole Blood}

The blood samples were collected, serum separated and stored in freezers at - 20 degree Celsius and then transported at 4 degree Celsius to the biotechnology department of Nitte Engineering College and the lead levels were determined by employing flame atomic absorption spectrometry. The results of B-Pb levels were expressed as $\mu \mathrm{g} / \mathrm{dl}$.

\section{Statistical Analysis}

Mean blood lead levels with standard deviation was be assessed. Data comparisons were performed by using student t-test and analysis of variance (ANOVA) with post hoc Tukey test. Linear regression was used to estimate the correlation between blood lead levels, systolic BP and diastolic BP. 
Logistic regression was used to estimate the correlation between blood lead and hypertension. Multivariate adjustment was done for co-variables and finally odds ratio was done for the correlation. Significance was set at P-value less than or equal to 0.05 . Ethical approval was obtained from the institutional ethics committee, K. S. Hegde Medical Academy.

\section{RESULTS}

A total of 50 cases and 50 healthy subjects (controls) fulfilling the criteria were included in this study. The general characteristics of this study with mean age in controls being
50.96 and in cases being 48.58. The mean systolic blood pressure among controls was 124.24 and cases were 150 . The mean diastolic blood pressure in controls was 78.2 and in cases were 93.68. The mean serum lead levels in controls was 16.94 and cases was 21.07 . In this study $52 \%$ of cases and controls were between the age group of 41 to 60 years. $22 \%$ of the subjects were more than 61 years of age.

In this study $45 \%$ of the total subjects were females and $55 \%$ were males. $46 \%$ of the controls and $44 \%$ of the cases were females. The mean serum lead levels in controls was 16.94 and in cases was 21.074 .

Comparison of the serum lead levels ( $\mathrm{mcg} / \mathrm{dl}$ ) between the cases and control showed that serum lead levels (mcg / dl) were higher in cases group with a t-value of -7.38 and was statistically significant with a P-value of $<0.001$.

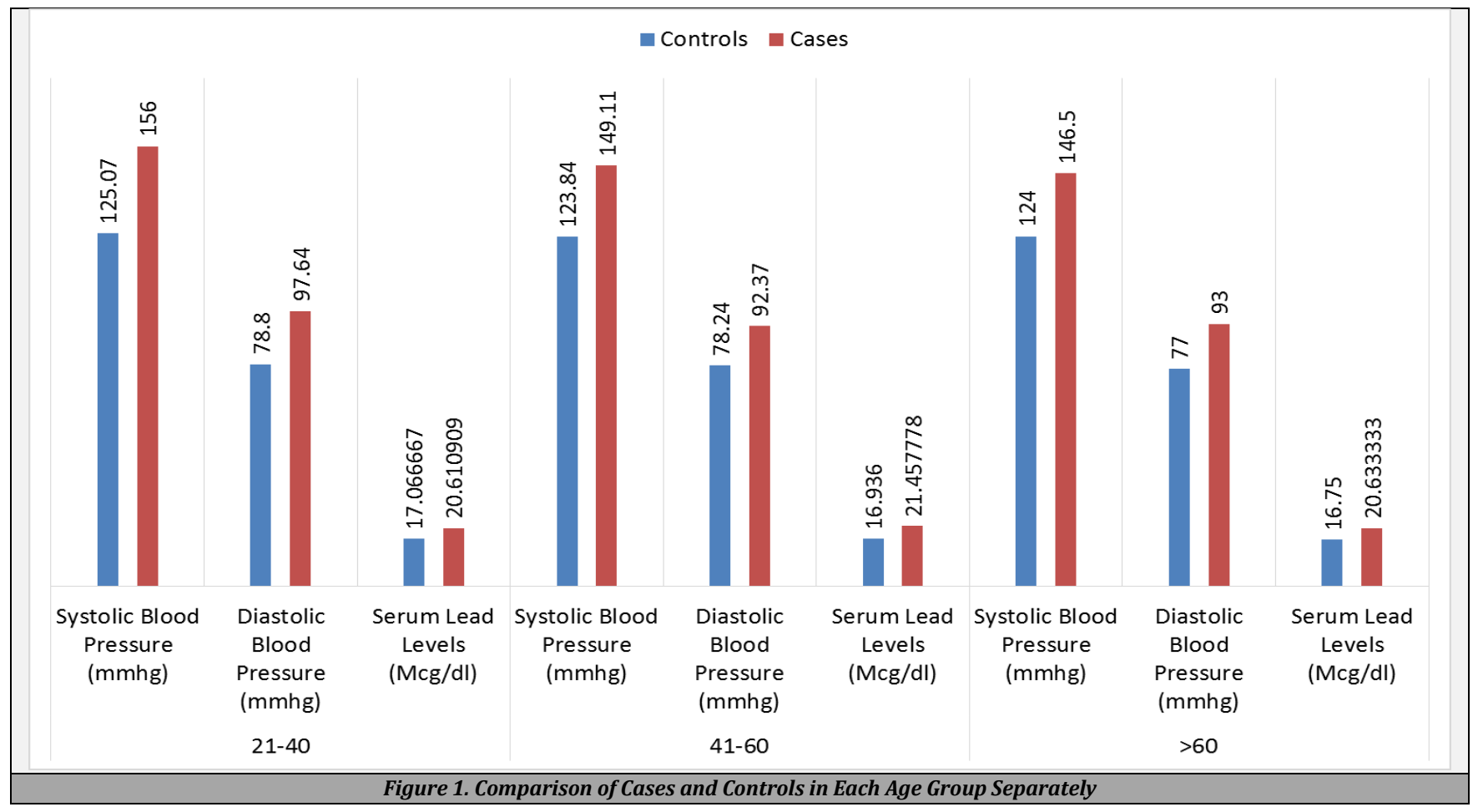

\begin{tabular}{|c|c|c|c|c|c|c|c|}
\hline \multirow{3}{*}{$\begin{array}{c}\text { Serum } \\
\text { Lead } \\
\text { Levels } \\
\text { (Mcg / dl) }\end{array}$} & Groups & $\mathbf{N}$ & Mean & $\begin{array}{c}\text { Std. } \\
\text { Deviation }\end{array}$ & $\begin{array}{l}\text { Statistics } \\
\text { / Mean } \\
\text { Squares }\end{array}$ & $\begin{array}{l}\text { df2 (Welch) } \\
\text { F (ANOVA) }\end{array}$ & $\begin{array}{l}\text { / } P \\
\text { Value }\end{array}$ \\
\hline & Stage 1 & 33 & 20.99394 & $\begin{array}{r}+723904 \\
+\end{array}$ & \multirow[b]{2}{*}{26.77} & \multirow[b]{2}{*}{33.975} & \multirow[b]{2}{*}{$\begin{array}{c}< \\
0.001\end{array}$} \\
\hline & Stage 2 & 17 & 21.22824 & 3.314537 & & & \\
\hline \multicolumn{8}{|c|}{ Table 1. Association of Stage of Hypertension and Serum Lead Levels } \\
\hline \multicolumn{8}{|c|}{$\begin{array}{l}\text { *Stage } 1 \text { Hypertension - SBP: - } 140-159 \mathrm{~mm} \backslash \mathrm{Hg} \text {, DBP: }-90 \text { - } 99 \mathrm{mmHg} \\
\text { * Stage } 2 \text { Hypertension - SBP: - = />160 mmHg, DBP: - = /> } 100 \mathrm{mmHg}\end{array}$} \\
\hline
\end{tabular}

\begin{tabular}{|c|c|c|c|c|c|}
\hline $\begin{array}{l}\text { Dependent } \\
\text { Variable }\end{array}$ & & & $\begin{array}{c}\text { Mean Difference } \\
(\mathrm{I}-\mathrm{J})\end{array}$ & Std. Error & P-Value \\
\hline \multirow{3}{*}{$\begin{array}{l}\text { Serum Lead } \\
\text { Levels (mcg / } \\
\text { dl) }\end{array}$} & \multirow{2}{*}{ Controls } & Stage 1 & $-4.0559394^{*}$ & 0.631368 & $<0.001$ \\
\hline & & Stage 2 & $-4.2902353^{*}$ & 0.790339 & $<0.001$ \\
\hline & Stage 1 & Stage 2 & -0.2343 & 0.840406 & 0.958 \\
\hline
\end{tabular}

The serum lead level in the age group 41 to 60 was higher compared to other age groups among cases (Figure 1). The serum lead levels in females were slightly higher compared to males in cases group.
Comparison of the serum lead levels ( $\mathrm{mcg} / \mathrm{dl}$ ) between males and females showed that serum lead levels (mcg / $\mathrm{dl}$ ) were higher in female group with a t-value of - 0.151 and was statistically non-significant with a P-value of 0.881 .

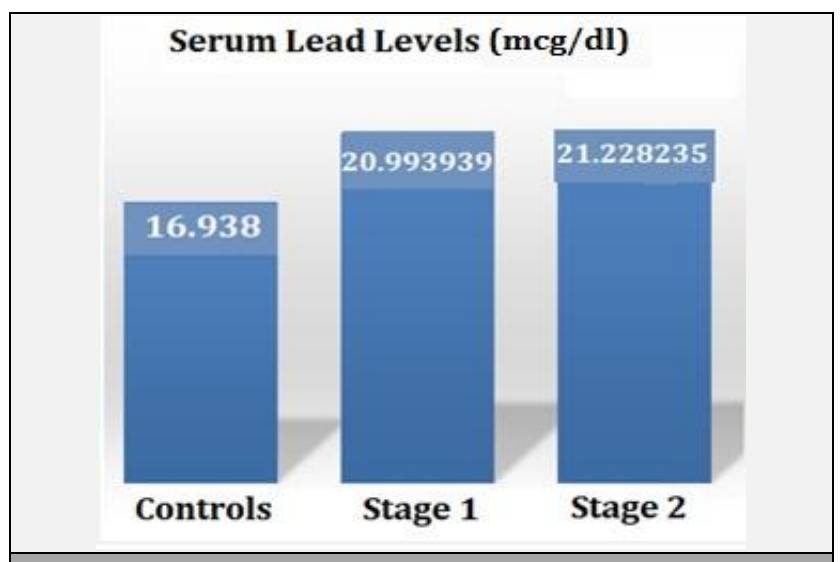

Figure 2. Correlation of Stage of Hypertension and Serum Lead Levels 


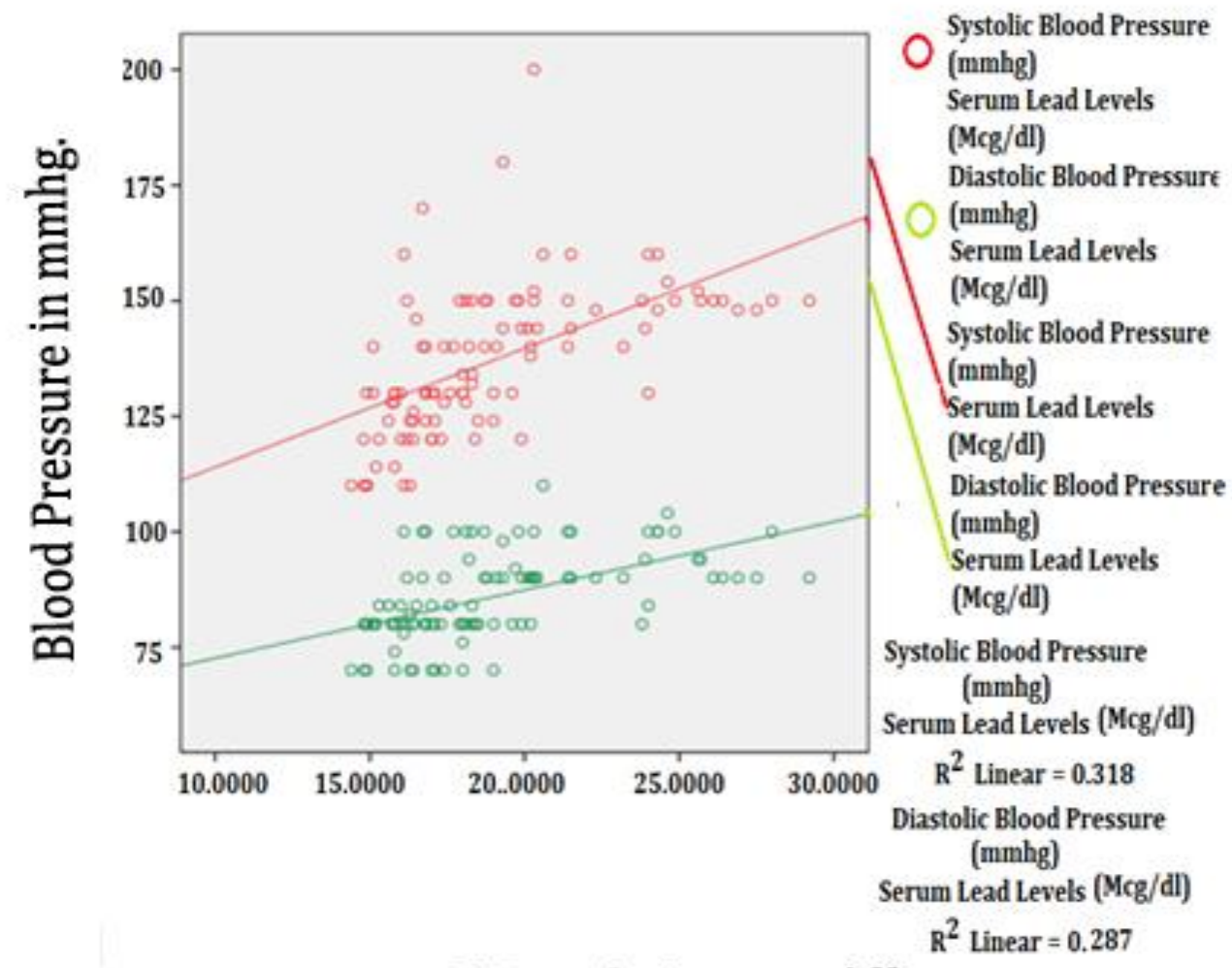

Serum Lead Levels in $\mathrm{mcg} / \mathrm{dl}$

Figure 3. Correlation of Lead with Systolic Blood Pressure and Diastolic Blood Pressure

Among the cases, 33 had stage 1 hypertension with a mean serum lead level of 20.99 and 17 of the cases had stage 2 hypertension with a mean serum lead level of 21.23 (Table 1).

Serum lead levels were mildly higher in stage 2 hypertensive patients compared to stage 1 (Figure 2).

Comparison of serum lead levels $(\mathrm{mcg} / \mathrm{dl}$ ) between the three groups showed that stage 2 group has the highest value of 21.228235 and controls had the least value of 16.938 . This difference was statistically significant with a test value of 33.975 and $p$ value of $<0.001$. Post hoc Tukey tests comparing controls and stage 1 groups showed a mean difference of 4.0559394* and was statistically significant with a P-value of $<0.001$. Comparing controls and stage 2 groups showed a mean difference of - 4.2902353* and was statistically significant with a P-value of $<0.001$. Comparing stage 1 and stage 2 groups showed a mean difference of -0.2342959 and was not statistically significant with a P-value of 0.958 (Table 2).

As systolic blood pressure and diastolic blood pressure increases the lead levels increases.

As lead level increased the systolic blood pressure increased which showed good correlation with a Pearson correlation of .564 and was statistically significant with a Pvalue of $<0.001$. As Lead level increased the diastolic blood pressure increased which showed very good correlation with a Pearson correlation of .766 and was statistically significant with a P-value of $<0.001$ (Figure 3 ).

It is predicted that for everyone mcg / dl rise in lead, systolic blood pressure increases by $2.573 \mathrm{mmHg}$ and is statistically significant.
It is predicted that for every one mcg / $\mathrm{dl}$ rise in lead, diastolic blood pressure increases by $1.478 \mathrm{mmHg}$ and is statistically significant.

\section{DISCUSSION}

From an epidemiologic perspective, there is no obvious level of blood pressure that defines hypertension. In adults, there is a continuous, incremental risk of cardiovascular disease, stroke and renal disease across levels of both systolic and diastolic blood pressure. ${ }^{1}$

Clinically, hypertension is defined as that level of blood pressure at which the institution of therapy reduces blood pressure-related morbidity and mortality. Current clinical criteria for defining hypertension generally are based on the average of two or more seated blood pressure readings during each of two or more outpatient visits. ${ }^{1}$

Hypertension is an iceberg disease in which a large percentage of a problem is subclinical, unreported or otherwise hidden from view. It is a growing concern worldwide with a rapidly increasing prevalence. Cardiovascular and cerebrovascular complications are known to be the leading cause of death among hypertensives. It is one of the major risk factors for cardiovascular mortality, which accounts for $20-50 \%$ of all deaths. ${ }^{5}$

High blood pressure (BP) is ranked third most important risk factor for attributable burden of disease in south Asia (2010). ${ }^{5}$ The burden of hypertension on cardiovascular health status and healthcare systems in India is substantial.6,7 $57 \%$ of 
all stroke deaths and $24 \%$ of all coronary heart disease (CHD) deaths in India are caused by hypertension. ${ }^{8} \mathrm{HTN}$ is one of the most important causes of premature death worldwide according to WHO. ${ }^{9}$ The global and regional burden of disease and risk factors study (2001), in a systematic analysis of population health data for attributable deaths and attributable disease burden, has ranked hypertension in South Asia as second after child underweight for age. ${ }^{10}$

In a worldwide data analysis for the global burden of HTN, $20.6 \%$ of Indian men and $20.9 \%$ of Indian women were suffering from HTN in 2005.11 The rates for HTN in percentage are projected to go up to 22.9 and 23.6 for Indian men and women, respectively by 2025.11 It is both a rural and urban health problem with a prevalence of $25 \%$ in urban and $10 \%$ in rural people in India.8,12-14 According to the WHO 2008 estimates, the prevalence of raised BP in Indians was $32.5 \%$ (33.2 \% in men and $31.7 \%$ in women). ${ }^{15}$ However, only about $25.6 \%$ of treated patients had their BP under control, in a multicentre study from India on awareness, treatment and adequacy of control of HTN. ${ }^{16}$

In this cross-sectional comparative study conducted at Justice K.S. Hegde Charitable Hospital, Mangalore, a total of 100 subjects were included in the study with 50 newly detected hypertensives and 50 healthy subjects, which were age and sex matched. Blood pressure and blood lead levels were measured, and their association was seen.

In our study, out of the total hundred subjects, majority of the cases were males ( $55 \%$ ) and between the age group 41 to 60 (52\%). Similar study conducted by Abdullah et al. ${ }^{17}$ on males showed a mean age of 39.92 in controls and 43.16 in cases.

In our study as the serum lead levels increased both diastolic and systolic blood pressures increased. A similar study done by Vupputuri et al. ${ }^{18}$ suggested that increase in levels of blood lead remain an important risk factor for elevated blood pressure. Many studies, but not all have indicated that environmental exposure to lead is associated with an increased risk of hypertension. Harlan ${ }^{19}$ in his study reported that both environmental and occupational exposure to lead has association with hypertension. Although the cardiovascular system is not typically taken as a primary target of lead toxicity, high concentrations of lead that might occur during occupational exposure are toxic to both the vascular smooth muscles and heart. ${ }^{20}$ Some reports suggested that, even transient exposure to lead during childhood can have a delayed and long term hypertensive effect. ${ }^{21}$ Studies done on experimental animals also supported a correlation between development of hypertension due to low level lead exposure.22,23 Martin et al. ${ }^{24}$ suggested that, lead due to recent doses has an acute effect on blood pressure and due to cumulative doses has a chronic effect on hypertension risk.

In our study, the blood lead levels were significantly higher in hypertensive patients than controls and had a positive correlation with the increase of systolic and diastolic blood pressure level. Similarly, Dursun et al. ${ }^{25}$ found a significant elevation in the mean systolic and diastolic blood pressures in the lead treated rats when compared to healthy controls. Robles et al. ${ }^{26}$ confirmed the hypertensive effect in rats to lead exposure. Vupputuri et al.18 also reported in black men significant associations between blood lead level and high systolic and diastolic blood pressures. Nash et al. ${ }^{27}$ found that, blood lead level is associated positively with both systolic and diastolic blood pressure in postmenopausal women. Recently, Kasperczyk et al.28 found that, blood lead levels were positively correlated with high arterial blood pressure (both systolic and diastolic). In another study, Afridi et al. ${ }^{29}$ reported higher levels of blood lead levels as well as a lower level of zinc, correlated well with the consequences of hypertension. Conversely, Staessen et al. ${ }^{30}$ did not find a significant relation between blood lead levels and hypertension.

Age is a very strong factor in the genesis of hypertension, but it seems to be independent of lead effect. A study done by Kasperczyk et al. ${ }^{28}$ did not find a correlation between blood lead levels and age. Our study showed a similar finding.

\section{Summary}

We conducted a cross sectional comparative study at a tertiary care hospital to assess the association of blood lead levels and elevated blood pressure in newly detected hypertensive patients. The subjects in both groups were age and sex matched. They had no previous history of hypertension or treatment for hypertension. Blood pressure values were measured and the blood lead levels of all the subjects were estimated.

We found that the blood lead level was significantly and positively correlated with blood pressure among newly detected hypertensive patients. Increase in blood lead levels caused increase in both systolic and diastolic blood pressures.

The mean systolic blood pressure in cases was $150 \mathrm{mmHg}$ and controls was $124.24 \mathrm{mmHg}$. The mean diastolic blood pressure in cases was $93.7 \mathrm{mmHg}$ and in controls was 78.2 mmHg. The mean lead levels of males in newly detected hypertensive cases was $21 \mathrm{mcg} / \mathrm{dl}$ and mean among controls was $16.94 \mathrm{mcg} / \mathrm{dl}$. The mean lead levels of females in newly detected hypertensive cases was $21.16 \mathrm{mcg} / \mathrm{dl}$ and mean among controls was $16.97 \mathrm{mcg} / \mathrm{dl}$. Stage 2 group had a higher serum lead level value of 21.228 compared to stage 1 and controls. Serum lead levels ( $\mathrm{mcg} / \mathrm{dl}$ ) were higher in females compared to males.

No significant change was found across age groups comparing blood lead levels and blood pressure. There was an increase in blood lead levels in hypertensive patients compared to the control group. No significant difference in the lead levels between stages of hypertension but marginally higher in stage 2. As lead level increased the systolic blood pressure increased which showed good correlation with a Pearson correlation of .564 and was statistically significant with a P-value of $<0.001$. As lead level increased the diastolic blood pressure increased which showed very good correlation with a Pearson correlation of .766 and was statistically significant with a P-value of $<0.001$. For every one mcg / dl rise in lead, systolic blood pressure increased by $2.573 \mathrm{mmHg}$ and was statistically significant. For every one mcg / dl rise in lead, diastolic blood pressure increased by $1.478 \mathrm{mmHg}$ and was statistically significant.

More similar studies are needed, especially in the field of metal exposure induced hypertension, to determine the relationship, their clinical significance, and the underlying mechanisms. Early identification of elevated lead levels can help in decreasing the overall morbidity and mortality associated with hypertension. 


\section{CONCLUSIONS}

The assessed blood lead levels in a newly detected hypertensive causes increase in both systolic and diastolic blood pressures. The blood lead level is significantly and positively correlated with blood pressure among newly detected hypertensive.

Data sharing statement provided by the authors is available with the full text of this article at jemds.com.

Financial or other competing interests: None.

Disclosure forms provided by the authors are available with the full text of this article at jemds.com.

\section{REFERENCES}

[1] Kasper DL, Fauci AS, Braunwald E, et al. Harrison's Principles of Internal Medicine. Vol. 298. 19th edn. New York: McGraw-Hill Publication 2015: p. 1611-20.

[2] Prozialeck WC, Edwards JR, Nebert DW, et al. The vascular system as a target of metal toxicity. Toxicol Sci 2008;102(2):207-18.

[3] Wu WT, Tsai PJ, Yang YH, et al. Health impacts associated with the implementation of a national petrol-lead phaseout program (PLPOP): evidence from Taiwan between 1981 and 2007. Sci Total Environ 2011;409(5):863-7.

[4] WHO. Global health risks: mortality and burden of disease attributable to selected major risks. Geneva: World Health Organization 2009. http://www.who.int/healthinfo/global_burden_disease/ GlobalHealthRisks_report_full.pdf

[5] Lim SS, Vos T, Flaxman AD, et al. A comparative risk assessment of burden of disease and injury attributable to 67 risk factors and risk factor clusters in 21 regions, 1990 2010: a systematic analysis for the Global Burden of Disease Study 2010. Lancet 2012;380(9859):2224-60.

[6] Leeder S, Raymond S, Greenberg H, et al. A race against time: the challenge of cardiovascular disease in developing economies. New York: Columbia University 2004.

[7] Reddy KS, Shah B, Varghese C, et al. Responding to the threat of chronic diseases in India. Lancet 2005;366:1744-9.

[8] Gupta R. Trends in hypertension epidemiology in India. J Hum Hypertens 2004;18(2):73-8.

[9] Mackay J, Mensah G. Atlas of Heart disease and stroke. Geneva: World Health Organization 2004.

[10] Lopez AD, Mathers CD, Ezzati M, et al. Global and regional burden of disease and risk factors, 2001: systematic analysis of population health data. Lancet 2006;367(9524):1747-57.

[11] Kearney PM, Whelton M, Reynolds K, et al. Global burden of hypertension: analysis of worldwide data. Lancet 2005;365(9455):217-23.

[12] Thankappan KR, Sivasankaran S, Sarma PS, et al. Prevalence-correlates-awareness-treatment and control of hypertension in Kumarakom, Kerala: baseline results of a community-based intervention program. Indian Heart J 2006;58(1):28-33.
[13] Gupta R. Meta-analysis of prevalence of hypertension in India. Indian Heart J 1997;49(1):450.

[14] Das SK, Sanyal K, Basu A. Study of urban community survey in India: growing trend of high prevalence of hypertension in a developing country. Int J Med Sci 2005;2(2):70-8.

[15] Non-communicable diseases country profiles 2011. http://www.who.int/nmh/countries/ind_en.pdf Accessed 10 May 2013.

[16] Hypertension Study Group. Prevalence, awareness, treatment and control of hypertension among the elderly in Bangladesh and India: a multicentre study. Bull World Health Organ 2001;79(6):490-500.

[17] Alghasham AA, Meki ARMA, Ismail HAS. Association of blood lead level with elevated blood pressure in hypertensive patients. Int J Health Sci (Qassim) Jan 2011;5(1):17-27.

[18] Vupputuri S, He J, Muntner P, et al. Blood lead level is associated with elevated blood pressure in blacks. Hypertension 2003;41(3):463-8.

[19] Harlan WR. The relationship of blood lead levels to blood pressure in the U.S. population. Environ Health Perspect 1988;78:9-13.

[20] Prozialeck WC, Edwards JR, Nebert DW, et al. The vascular system as a target of metal toxicity. Toxicol Sci 2008;102(2):207-18.

[21] Hu H. A 50-year follow-up of childhood plumbism. Hypertension, renal function and hemoglobin levels among survivors. Am J Dis Child 1991;145(6):681-7.

[22] Khalil-Manesh F, Gonick HC, Weiler EW, et al. Leadinduced hypertension: possible role of endothelial factors. Am J Hypertens 1993;6(9):723-9.

[23] Vaziri ND, Liang K, Ding Y. Increased nitric oxide inactivation by reactive oxygen species in lead-induced hypertension. Kidney Int 1999;56(4):1492-8.

[24] Martin D, Glass TA, Bandeen-Roche K, et al. Association of blood lead and tibia lead with blood pressure and hypertension in a community sample of older adults. Am J Epidemiol 2006;163(5):467-78.

[25] Dursun N, Arifoglu C, Süer C, et al. Blood pressure relationship to nitric oxide, lipid peroxidation, renal function and renal blood flow in rats exposed to low lead levels. Biol Trace Elem Res 2005;104(2):141-9.

[26] Robles HV, Romo E, Sanchez-Mendoza A, et al. Lead exposure effect on angiotensin II renal vasoconstriction. Hum Exp Toxicol 2007;26(6):499-507.

[27] Nash D, Magder L, Lustberg M, et al. Blood lead, blood pressure and hypertension in perimenopausal and postmenopausal women. JAMA 2003;289(12):1523-32.

[28] Kasperczyk S, Kasperczyk J, Ostałowska A, et al. The role of the antioxidant enzymes in erythrocytes in the development of arterial hypertension among humans exposed to lead. Biol Trace Elem Res 2009;130(2):95106.

[29] Afridi HI, Kazi TG, Kazi NG, et al. Evaluation of cadmium, lead, nickel and zinc status in biological samples of smokers and nonsmokers hypertensive patients. J Hum Hypertens 2010;24(1):34-43.

[30] Staessen JA, Lauwerys RR, Buchet JP, et al. Impairment of renal function with increasing blood lead concentrations in the general population. The Cadmibel Study Group. N Engl J Med 1992;327(3):151-6. 